\title{
Strong-field gravitational-wave emission in Schwarzschild and Kerr geometries: some general considerations
}

\author{
J. F. Rodríguez ${ }^{1,2, \star}$, J. A. Rueda ${ }^{1,2, \star \star}$, and R. Ruffini ${ }^{1,2, \star \star \star}$ \\ ${ }^{1}$ ICRANet, Piazza della Repubblica 10, I-65122 Pescara, Italy \\ ${ }^{2}$ Dipartimento di Fisica and ICRA, Sapienza Università di Roma, P.le Aldo Moro 5, I-00185 Rome, Italy
}

\begin{abstract}
We have used the perturbations of the exact solutions of the Einstein equations to estimate the relativistic wave emission of a test particle orbiting around a black hole. We show how the hamiltonian equations of motion of a test particle augmented with the radiation-reaction force can establish a priori constraints on the possible phenomena occurring in the merger of compact objects. The dynamical evolution consists of a helicoidal sequence of quasi-circular orbits, induced by the radiation-reaction and the background spacetime. Near the innermost stable circular orbit the evolution is followed by a smooth transition and finally plunges geodesically into the black hole horizon. This analysis gives physical insight of the merger of two equal masses objects.
\end{abstract}

\section{Introduction}

The problem of the merger of two massive objects is too complicated to be directly analyzed by solving the full Einstein equations. One reasonable attempt is to limit to the case of perturbations in the strong-field limit. We are going to neglect all post-Newtonian approaches to this problem. There are idealized processes in the strong-field limit to describe the motion of a test particle plunging into the field of an already formed black hole $(\mathrm{BH})$. One case is purely geodesic where a test particle initially at rest or with a finite kinetic energy plunges from an infinite distance. Broadly speaking, all particles starting from infinite distance give rise to "a precursor, a main burst and a ringing tail". A finite energy emission occurs, $\Delta E=\eta m(m / M)$, where $m$ and $M$ are the particle and BH mass, with $\eta$ ranging from 0.01 to almost 1 , as a function of the nature of the $\mathrm{BH}$ and of the initial energy and angular momentum of the particle [1-4]. Another case, on which we focus here, it is analyzed by the study of the hamiltonian equations augmented with radiative forces. It consists of a "helicoidal" sequence of quasi-circular orbits, mainly driven by the radiation-reactionin the early stages. It follows a smooth transition near the innermost stable circular orbit (ISCO), and from this point it plunges describing a pure geodesic of the background spacetime. In Sec. 2 we recall the results on the case of circular orbits without taking into account radiation reaction and find the gravitational-wave energy and angular momentum flux at infinity, following the Sasaki-Nakamura method. In Sec. 3 we stufy the evolution of the particle, taking into account the radiation-reaction, obtaining the "helicoidal drifting sequence" passing through the last circular orbit and finally plunging.

\footnotetext{
^e-mail: jose.rodriguez@icranet.org

$\star \star$ e-mail: jorge.rueda@icra.it

$\star \star \star$ e-mail: ruffini@icra.it
} 

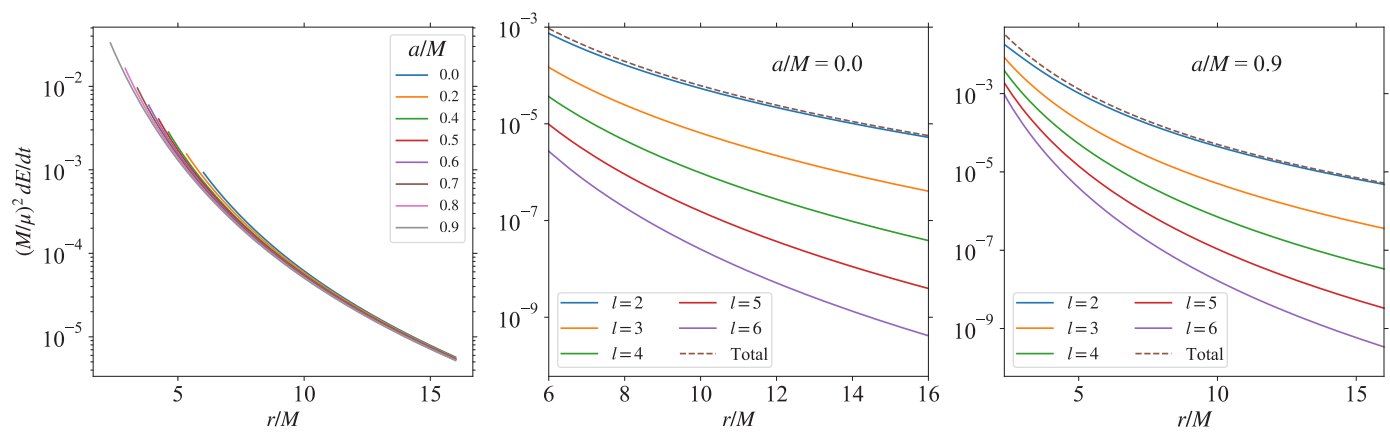

Figure 1. The figure on the left shows the total gravitational-wave energy flux at infinity, $\dot{E} \equiv d E / d t$, emitted by the test particle in circular orbits around the BH for different values of the normalized spin, as a function of $r / M$. The center and right figures show the contribution of the gravitational-wave modes $l \geq 2$ to the total gravitational-wave energy flux at infinity in the case a Schwarzschild BH and a Kerr black hole with $a / M=0.9$, correspondingly.

\section{Gravitational Radiation Fluxes for Circular Orbits}

The calculation of the energy and momentum fluxes due to a particle orbiting a BH can be done by using standard metric perturbation theory, e.g. the work of Regge-Wheeler for perturbations in the Schwarzschild spacetime. Nevertheless, it is convenient to use an alternative approach developed by Teukolsky $[5,6]$ which deals with curvature perturbations. After performing a variable separation, it is possible to solve the corresponding equations. Unfortunately, the Teukolsky radial equation has a long-range potential and its numerical integration with boundary conditions is difficult. Sasaki and Nakamura [7,8] found a change of variables that introduces a short-range and well-behaved potential $U(r)$. The Sasaki-Nakamura equation is:

$$
X_{l m \omega}^{\prime \prime}-F(r) X_{l m \omega}^{\prime}-U(r) X_{l m \omega}=\mathcal{S}_{l m \omega} .
$$

Details on the functions $F, U$ and $\mathcal{S}_{l m \omega}$ and their numerical solution can be found in Refs. [8] and [9]). The solution must satisfy the boundary conditions of out-going radiation at $\infty$ and in-going radiation at the horizon. To solve the radial equation with this suitable boundary conditions we used the Green's function technique. The procedure we used was the following: first, we solved numerically the Eq. (1) with the suitable boundary conditions, and then inverted the transformation to find the original radial function. Since we have used the Green's function technique, there are two solutions of the homogeneous Sasaki-Nakamura equation. The solution which satisfies the boundary condition at the outer horizon is $X_{\operatorname{lm} \omega}^{H}$ and the solution that satisfies the boundary condition at $\infty$ is $X_{\operatorname{lm} \omega}^{\infty}$. In the case of circular orbits, the energy and angular momentum fluxes carried by the gravitational waves to infinity are given by

$$
\begin{aligned}
& \frac{d E}{d t}=\sum_{l \geq 2}^{\infty} \sum_{m=-l}^{l} \frac{\left|Z_{l m \omega}^{H}\right|^{2}}{4 \pi \omega_{m}^{2}}, \\
& \frac{d J}{d t}=\sum_{l \geq 2}^{\infty} \sum_{m=-l}^{l} \frac{m\left|Z_{l m \omega}^{H}\right|^{2}}{4 \pi \omega_{m}^{3}},
\end{aligned}
$$




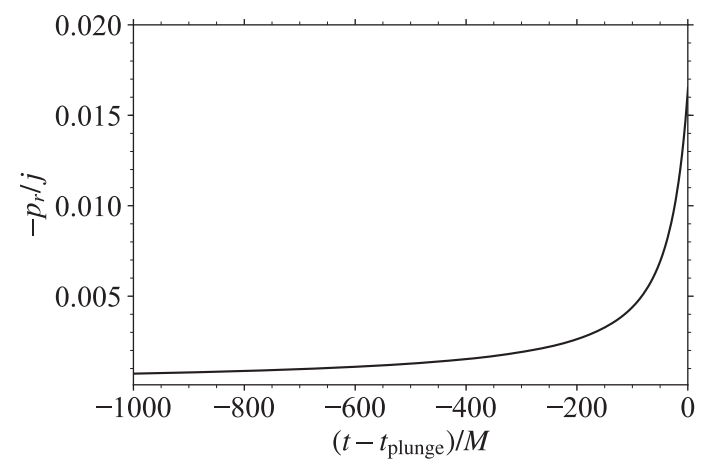

Figure 2. $p_{r} / j$ : ratio of the radial momentum to the angular momentum per unit mass $p_{\phi} / \mu$ of a test particle in the helicoidal drifting sequence around a Kerr black hole with $a / M=0.7$. The mass-ratio is $\mu / M=1 / 100$. The plot shows the ratio from $r \approx 5.32 M$ up to the location of the last circular orbit at $r \approx 3.39 M$. The time $t_{\text {plunge }}$ is here defined as the time of the passage of the particle through the ISCO.

where $\omega_{m}=m \omega$, and $Z_{l m \omega}^{H}$ is a complex number found from $X_{l m \omega}^{H}$ (see eg. [9] for the explicit expression). It turns out that $Z_{\operatorname{lm} \omega}^{H}$ is a function of the orbital frequency $\Omega$. The results for different values of the normalized spin of the $\mathrm{BH}$ are shown in Fig. 1. We found agreement with the results of Refs. $[10,11]$.

\section{Helicoidal drifting sequence}

After calculating the gravitational radiation induced by a particle in a circular orbit, we proceed to find the dynamical effect in the particle's trajectory. In order to calculate this we used the Hamiltonian formalism augmented with a non-conservative force coming from the radiation. The Hamiltonian of the test particle of mass $\mu$ in the field of the Kerr BH of mass $M$ is given by (see e.g. [12], and references therein)

$$
H=-p_{t}=-N^{i} p_{i}+N \sqrt{\mu^{2}+\gamma^{i j} p_{i} p_{j}},
$$

where $N=1 / \sqrt{-g^{00}}, N^{i}=-g^{t i} / g^{t t}$ and $\gamma^{i j}=g^{i j}+N^{i} N^{j} / N^{2}=g^{i j}-g^{t i} g^{t j} / g^{t t}$. Here Latin index stands for the spatial Boyer-Lindquist coordinates $(r, \theta, \phi), p_{r}$ and $p_{\phi}$ are, respectively, the radial and the angular momentum of the particle. The Hamilton canonical equations on the equatorial plane $\theta=\pi / 2$ under the action of radial and azimuthal dissipative effects can then be written as (for a review on the subject see e.g. [13] in the extreme mass-ratio limit)

$$
\frac{d r}{d t}=\frac{\partial H}{\partial p_{r}}, \quad \frac{d \phi}{d t} \equiv \Omega=\frac{\partial H}{\partial p_{\phi}}, \quad \frac{d p_{r}}{d t}=-\frac{\partial H}{\partial r}+\mathcal{F}_{r}^{\mathrm{nc}}, \quad \frac{d p_{\phi}}{d t}=\mathcal{F}_{\phi}^{\mathrm{nc}},
$$

We adopt for the radial and azimuthal non-conservative radiation-reaction forces:

$$
\begin{aligned}
& \mathcal{F}_{r}^{\mathrm{nc}}=0, \\
& \mathcal{F}_{\phi}^{\mathrm{nc}}=-\frac{d J}{d t} .
\end{aligned}
$$



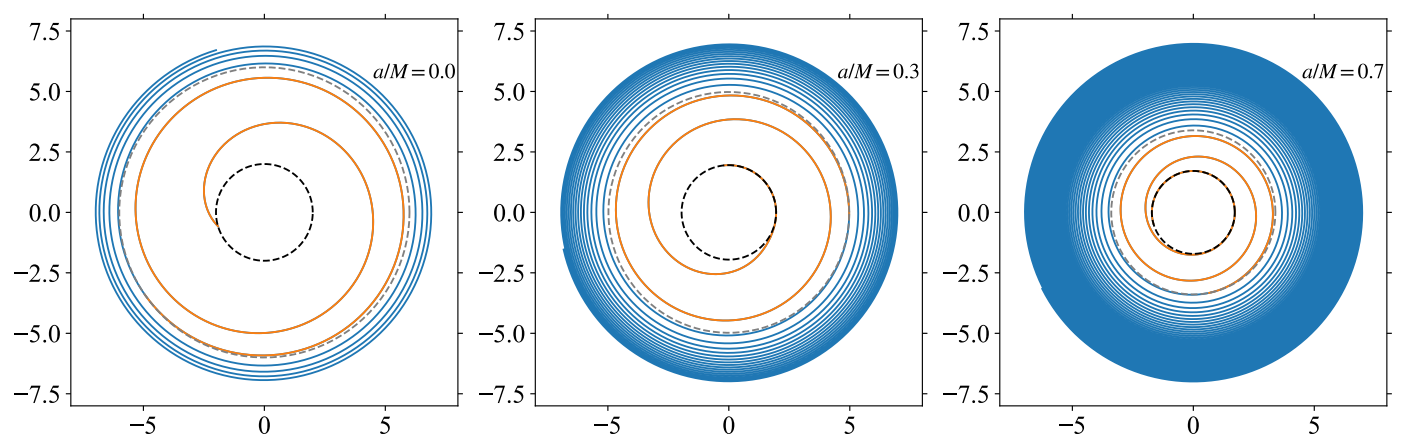

Figure 3. Trajectory of a test particle in the helicoidal drifting sequence around a Schwarzschild black hole, $a / M=0$ (left panel) and around a Kerr black hole with $a / M=0.3$ (center panel) and with $a / M=0.7$ (right panel). The blue part of the trajectory goes from $r=7 M$ up to the location of the corresponding ISCO (graydashed circle), while the orange color indicates the plunge regime. The black-hole horizon is indicated with a black-dashed circle. The mass-ratio is $\mu / M=1 / 100$.

where $J$ is the angular momentum carried out to infinity by the gravitational waves given by Eq. (3). The assumption (6) is supported from the results of [14] in which it can be seen that the linear momentum carried out by the waves to infinity satisfies

$$
\frac{d p_{r}^{\mathrm{GW}}}{d t} \ll\left|\frac{\partial H}{\partial r}\right| .
$$

We have checked from our numerical computations that $|1-\Omega(d J / d t) /(d E / d t)| \sim 10^{-6}$ during the evolution.

In the traditional literature the evolution of the particle has been treated "adiabatically" . Within this formalism, the test particle that it is initially on a stable circular orbit, evolves to another circular orbit by the emission of gravitational radiation. This approach relies on the assumption that the radial velocity $\dot{r}$ is much less that the azimuthal velocity $r \Omega$. The adiabatic condition breaks down near the ISCO. On the other hand, in our formalism there is an additional effect induced by the radial momentum $p_{r}$. During the early stages of the evolution, the two approaches are practically equivalent. This is due to the fact that during this phase the radial momentum is negligible. However, near the ISCO $p_{r}$ becomes appreciable (see Fig. 2). This fact allows a smooth transition through the ISCO. Since the radiation-reaction was calculating by assuming circular orbits, after the ISCO it can not be used. After this point we turn off the gravitational-reaction force and the test particles follows a pure geodesic down to the horizon. It is worthwhile to mention that when the particle arrives at $r=r_{\text {ISCO }}$, the energy and angular momentum are different from the corresponding values of the ISCO. Furthermore, as already mentioned the radial momentum is different from zero as opposed to the adiabtic approach. All this induces the particle to plunge into the black even without radiating. The Fig. 3 shows the numerical results of the formalism for selected values of the normalized spin of the $\mathrm{BH}$.

\section{Conclusions}

The case of a particle starting from a finite radius in a quasi-circular orbit leads to a novel "helicoidal drifting sequence", leading finally to a smooth merging into the black hole. There is a smooth transi- 
tion in the Schwarzschild and Kerr cases. After the particle crosses the ISCO the trajectory is purely geodesic. When the radiation reaction is taken into account, as evidenced in Fig. 3, there is a clear appearance of "helicoidal drifting sequence". The more the particle gets closer to the horizon the more is the particle drift due to the increasing contribution of the radial momentum (see Fig. 2). The ISCO in this context does not witness any special role and only assists to the passage of the plunging particle in view of the acquired radial momentum in its previous history. The final result is the one of a test particle smoothly merging in the Kerr black hole without any burst.

\section{References}

[1] M. Davis, R. Ruffini, W.H. Press, R.H. Price, Physical Review Letters 27, 1466 (1971)

[2] S.L. Detweiler, E. Szedenits, Jr., ApJ231, 211 (1979)

[3] R. Ruffini, M. Sasaki, Progress of Theoretical Physics 66, 1627 (1981)

[4] Y. Kojima, T. Nakamura, Progress of Theoretical Physics 71, 79 (1984)

[5] S.A. Teukolsky, ApJ185, 635 (1973)

[6] S.A. Teukolsky, W.H. Press, ApJ193, 443 (1974)

[7] M. Sasaki, T. Nakamura, Progress of Theoretical Physics 67, 1788 (1982)

[8] M. Sasaki, T. Nakamura, Physics Letters A 89, 68 (1982)

[9] S.A. Hughes, Phys. Rev. D61, 084004 (2000), gr-qc/9910091

[10] S.L. Detweiler, ApJ225, 687 (1978)

[11] R. Fujita, H. Tagoshi, Progress of Theoretical Physics 112, 415 (2004), gr-qc/0410018

[12] R.T. Jantzen, P. Carini, D. Bini, Annals of Physics 215, 1 (1992), gr-qc/0106043

[13] W.B. Han, Z. Cao, Phys. Rev. D84, 044014 (2011), 1108.0995

[14] M.J. Fitchett, S. Detweiler, MNRAS211, 933 (1984) 